\title{
Management of infected nonunion of long bones: The last decade (1996-2006)
}

\author{
N.S. Motsitsi \\ University of Pretoria, Department of Orthopaedic Surgery, Kalafong Hospital, Private Bag X396, \\ Pretoria 0001, South Africa
}

\section{Summary}

Infected nonunion is one of the most challenging orthopaedic complications to manage. There is considerable morbidity associated with infected nonunion. There is significant impact on the life of the patient; social, financial, physical, and mental. Infected nonunion may be a limb-threatening complication.

Significant progress has been made in the management of infected nonunion in the last decade. There are clear guidelines for pre-operative evaluation and treatment strategy. The recommended strategy is the 'infection-elimination first' strategy. This strategy involves two steps: control of infection by local radical debridement of dead tissue followed by reconstruction. There are four operative techniques (with considerable overlap among them) which have been used in the past decade: llizarov, intramedullary devices with or without the use of external fixator, free tissue transfer, and in situ reconstruction.

Bone results are, in general, better compared to functional results. Overall, the outcome following treatment of infected nonunion are good to excellent.

Keywords: Infected nonunion; Long bones; Management; Outcome; Last decade

\section{Introduction}

Infected nonunion is a formidable complication to treat. It is a complex problem with considerable morbidity and can threaten the life and limb of the patient. ${ }^{7}, 27-28$ The mortality rate from osteitis in the pre-antibiotic era was $27-30 \% .{ }^{27}$ There is considerable social, financial, physical, and psychological impact on the patient. Limb loss due to chronic osteitis is significant: $90 \%$ in B-host. ${ }^{14}$

Great progress has been made in the management of infected nonunion in the past two decades. ${ }^{16}$ There has been significant progress in the areas of patients' clinical evaluation, pre-operative diagnostic work-up, treatment philosophy, and patient outcome. The purpose of this article is to review published management strategies in the treatment of infected nonunion in the past decade. 


\section{Discussion}

Approach to a patient who presents with a clinical problem of infected nonunion must be logical. The surgeon must have a plan made up of the following components: clinical evaluation, diagnostic work-up, and treatment strategy.

\section{Clinical evaluation}

Clinical evaluation is the first and one of the most important steps in the management of a patient with infected nonunion. What does a surgeon evaluate The surgeon must perform a thorough evaluation of the patient and the affected limb.

\section{The patient}

The surgeon must note the following: the history of the injury, previous operation(s), complications, expectations of the patient, the level of motivation, social and occupational history, medical history, and the impact of the condition on the life of the patient.

\section{The injured limb}

The following needs thorough evaluation: deformity, limb length discrepancy, the presence or absence of a sinus or sinuses, signs of active infection, scarring due to previous surgery, neurological and vascular status, and the type of nonunion.

At the end of this evaluation, the clinician should be able to classify the patient in one of the following categories. ${ }^{5}$

1. Criteria for clinical infection.

- Open wound or draining sinus pre-operatively, or

- Frank pus found at operation.

2. Criteria for negative infection.

- No overt signs of infection pre-operatively.

- No history of open wound or multiple previous operations.

- No post-operative infection for at least 1 year after surgery.

-Where there is a cause other than sepsis to explain the nonunion. 


\section{Suspicious of infection.}

- History of open wound or multiple surgery.

- No other reason to develop nonunion.

Infection is a major cause of nonunion. ${ }^{5}$ Infection can be sub-clinical. It is mandatory to exclude infection in all cases of nonunion.

\section{Laboratory investigations}

These investigations are an integral part of the patient's work-up and they are commonly overlooked. They are cheap, easily available, and can be very informative. They are useful in diagnosis and monitoring the response during treatment, although they can be equivocal or negative particularly in sub-clinical infection.

\section{Haematological investigations}

Full blood count (FBC), C-reactive protein (CRP) and erythrocytes sedimentation rate (ESR) are the usual tests requested in infections. The white blood cell (WBC) count may be high, normal or borderline; depending on the activity of infection. All these parameters may be normal in sub-clinical infection. The ESR and CRP are helpful in monitoring progress during treatment.

\section{Pre-operative biopsy}

It is not clear from the literature whether it is mandatory to do biopsy in all cases of nonunion. It is good policy [it is a protocol in this author's Department] to exclude infection in all cases of nonunion. The source of infection can be soft tissue or bone. Multiple biopsies (three to five) must be sampled deep from nonunion site. At least two of the specimens must be positive to document infection. ${ }^{5}$ The culture results commonly yield multiple organisms: this has no influence in the treatment outcome. ${ }^{5}$

\section{Histological evaluation}

Histology has a sensitivity of $87.1 \%$ and $100 \%$ specificity. ${ }^{5}$ It is of value when microbiological results are negative or equivocal.

\section{Radiological investigations}

Radiological investigations focus on bone and soft tissue pathology. They delineate the pattern and extent of infection. The most commonly used investigations are; routine $\mathrm{XR}, \mathrm{MRI}, \mathrm{CT}$, and bone scan. 


\section{Plain radiology $(X R)$}

Good quality XR offers a tremendous amount of information. The type of osteomyelitis and its extent can be well defined by XR. The Cierny-Mader classification of osteomyelitis is based on routine XR. ${ }^{15}$ This classification forms the basis of treatment approach. According to this system of classification, infected nonunion is classified as type IV (diffuse type). If you add patient or host evaluation, the whole classification is complete: the host can be either A, B (locally or systemically compromised) or $\mathrm{C}$. The best treatment strategy is then planned accordingly. Domenico et al. also use routine XR to classify nonunion. ${ }^{2}$ This is a very simple system and has a bearing in the approach to patient's treatment: infected nonunion is classified as type 4 and needs biological treatment. XR tends to underestimate the extent of bone involvement: a crucial concept to understand.

\section{MRI and CT}

These investigations are also non-invasive. They are not routinely done. MRI is excellent in differentiating between bone and soft tissue infection: an important point to know before pre-operative biopsy. CT is excellent in delineating the extent and the pattern of bone involvement.

MRI has certain pitfalls: it does depend, to a certain extend, on how it was done. It needs the combination of T1, STIR, and T2 images. The combination of these images increases specificity from 75 to $82 \% .^{19}$ The sensitivity is $100 \%$. The CT is particularly useful in the evaluation of intramedullary osteitis. The two investigations tend to over-estimate the extent of bone involvement.

\section{Scintigraphic techniques}

These techniques are invasive and expensive compared to other modalities. Bone involvement in chronic osteomyelitis can be patchy: bone Scintigraphy is able to detect this pattern of infection. Patchy bone infection can be missed during biopsy and thus giving false negative results. There are many different types of Scintigraphy and each appropriate in certain clinical situation(s).

Commonly used bone scans are; Technetium scan, 111-Indium-IgG imaging, and 111-Indium-oxime WBC.

\section{Technetium bone scan}

99Tc-MDP scan is commonly used in the investigations of infections. The sensitivity is $100 \%$ and the specificity is $0 \% .{ }^{26}$ 


\section{1-Indium-IgG imaging}

It is a sensitive technique for the evaluation of infection and inflammatory joint disease. It has $93 \%$ specificity in chronic osteomyelitis. It has a high negative predictive value.

\section{1-Indium-oxime}

WBC scan with $99 \mathrm{~m}$ Tc-sulphur colloid bone marrow scan. This is the gold standard for the study of infection in total hip arthroplasty. It indicates bone marrow activity and thus can be useful in screening for bone necrosis. It can be a useful study in infections of the musculo-skeletal system. ${ }^{17}$

The above investigations inform the surgeon about the magnitude of the clinical problem so that he can make informed surgical decisions. Unfortunately, the extent of bone involvement can only be made intra-operatively.

\section{Treatment philosophy}

Treatment of infected nonunion is technically demanding, prolonged, and needs a team. There are two schools of thought in the treatment of infected nonunion $^{10,13}$ : the 'union-first' strategy and the 'infection-elimination first' strategy. The first strategy aims at achieving union first and then dealing with the problem of infection as the problem presents itself. This approach does not aim at eradication of infection as the main objective. The second strategy aims at elimination of infection as the first and major objective and bone union as the next objective.

The second strategy is the more popular one and has been extensively applied in the last decade. This strategy does have its drawback: bone debridement leads to a defect which dramatically increases the complexity of reconstruction. ${ }^{20}$ This strategy must be approached with great care because it requires very experienced surgeons: it is a procedure for the highly skilled. The strategy has two steps: radical debridement of necrotic tissue (bone and soft tissue) followed by reconstruction of bone (to effect union) plus soft tissue (to provide healthy viable coverage). The bone defect (dead space) that results from bone debridement can be managed by antibiotic-impregnated bone cement. Once infection is eradicated or controlled, the second stage is commenced.

There are four surgical techniques which have been in use and published in the last decade. There is a great degree of overlap in these techniques. The techniques are: the llizarov, intramedullary device with or without external fixator, free tissue transfer, and in situ reconstruction. 


\section{The Ilizarov technique}

This technique is regarded by many surgeons as a standard treatment in infected nonunion. It is a salvage operation, ${ }^{3}$ suitable for highly selected and motivated patients. The technique can address many problems simultaneously: bone defects, deformity, malrotation, limb-length discrepancy, and nonunion. ${ }^{9}$ It is the most reliable technique for regaining a stable and functional leg. There are over sixty patients treated with this technique and reported in the literature in the last decade. There are different ways of using the llizarov in infected nonunion;

If there is no bone defect, the nonunion can be compressed and the position maintained until union. Infection is not eradicated or addressed.

If there is bone defect initially or after bone debridement, there are two options: acute shortening or bone transport.

Acute shortening is followed by limb lengthening to restore length. ${ }^{20}$ Acute shortening causes arteriolar occlusion and formation of scar tissue, which may cause difficulty with later limb length restoration.

Bone transport is a very good technique for closure of large bone defects. The advantage of this procedure is that there is simultaneous soft tissue transport, but the disadvantage is that the transported soft tissue is atrophic and makes a poor soft tissue envelope. ${ }^{20}$ This type of soft tissue coverage is not ideal for infected non-union as the soft tissue coverage over the docking site may be thin and hypersensitive. ${ }^{25}$ Soft tissue closure can also be achieved by skin traction during bone transport. The llizarov technique can be successfully applied in children with very good results. ${ }^{23}$

The results with the llizarov are excellent. ${ }^{6,9}$ Overall, bone results are good to excellent in $80 \%$ and functional results are good to excellent in $66.7 \%$. ${ }^{9}$ The functional results are, as a general rule, poorer compared to bone results. ${ }^{4}$ Functional results depend on the extent of damage sustained by soft tissue at the time of injury and or during subsequent surgical interventions.

The complication rate associated with the use of llizarov technique (both minor and major) may reach $87 \%$. It must be borne in mind that these patients are neglected cases with multiple unsuccessful previous operations, and it is not easy to decide whether these patients' limbs must be amputated or salvaged.

\section{Intramedullary devices with or without external fixators}

Bone transport over an intramedullary device is a relatively new technique in the treatment of infected nonunion. The advantages of this technique are; fracture stabilisation, prevention of limb shortening and malrotation, and early partial weight-bearing. ${ }^{8}$ There are 43 reported patients who have been treated with this 
type of operative technique. ${ }^{8,18}$ This technique still needs to be evaluated. Internal fixation (plating) has not been reported in the management of infected nonunion of long bones. ${ }^{21}$

\section{Free tissue transfer}

Free tissue transfer (bone, soft tissue or both) are technically demanding, and require close co-operation between plastic and orthopaedic surgeons. Options for vascularised bone are; fibula, iliac crest, ribs, and scapula. Cheng et al. ${ }^{13}$ used vascularised two ribs graft plus serratus anterior in six patients with infected nonunion. All fractures united and infection was controlled, but there were two stress fractures of the implanted ribs. The fractures were treated non operationally and they united. Vascularised bone and tissue can be used in difficult infections. Yasunori et al. ${ }^{24}$ used fibular grafts in infected nonunion caused by Methicillin-Resistant Staphylococcus Aureus (MRSA), 20, obtaining union in $18 / 20^{90 \%}$. Infection was controlled using the two-stage strategy.

The two-stage strategy provides a biological environment which is necessary for healing of bone and soft tissue.

\section{Debridement, bone-grafting, and soft tissue coverage (in situ reconstruction)}

Not all infected nonunions need complicated procedures. In situ reconstruction does offer an alternative. Small defects $(2-4 \mathrm{~cm})$ can be adequately treated with simpler techniques, using the same two-stage strategy. Cancellous bone-grafting (with or without antibiotics) is performed in the second stage. ${ }^{10-12}$ Soft tissue coverage can be obtained by either local or vascularised muscle flap. Antibiotics mixed with cancellous bone during the second stage provides high local concentration of the antibiotics for up to 3 weeks without systemic signs of antibiotic toxicity. The effect(s) of mixing antibiotics with cancellous bone grafting (in terms of bone union or incorporation) is not known at this stage. The technique is very effective in the eradication of infection: up to $94.4 \%$ success rate. $^{10,16}$

In situ fibular transfer has been successfully used in children with tibial infected nonunion. Haluk et al. ${ }^{1}$ used this technique in four children. He obtained union in all and infection was successfully eradicated. The angular deformity that occurs so commonly after this procedure will correct spontaneously in the majority of children. Vascularised fibular transfer can also be performed in children, without affecting growth of the donor leg. ${ }^{22}$ 


\section{Conclusion}

Published literature (in the last decade) on the management of infected nonunion has provided the following guidelines:

- Not every patient with infected nonunion of long bone is a candidate for reconstruction.

- A philosophy of 'infection-elimination first' is a definite way of eradicating or controlling infection.

- A two-stage strategy is the best and well-proven one.

\section{Conflict of interest}

No conflict of interest.

\section{References}

1. H. Agus, M. Arslantas, O. Kalenderer and I.T. Ozcalabi, Treatment of infected pseudoarthrosis of the tibia by in situ fibular transfer in children., Injury Int J Care Injured 36 (2005), pp. 1476-1479.

2. D. Aloj, A. Biasebetti and G. Di Gregorio et al., Mechanical and biological treatment of long bones nonunion, Injury Int J Care Injured 365 (2005), pp. S45S50.

3. Z.M. Arnez and D. Smrke, Treatment of extensive bone and soft tissue defects of the lower limb by traction and free-flap transfer, Injury Int J Care Injured 31 (2000), pp. 153-162.

4. A.S. Atesalp, M. Basbozkurt, M. Komurcu and M. Kurklu, The treatment of infected tibial nonunion with aggressive Debride'ment and internal bone transport, Mil Med 167 (12) (2002), pp. 978-981.

5. N.A. Athanasou, A.H.R. Simpson and M.K. Wood, Histological assessment of the presence or absence of infection in fracture nonunion, Injury Int J Care Injured 33 (2002), pp. 151-155.

6. V. Barbarossa, B.R. Matkovic and N. Vucic et al., Treatment of osteomyelitis and infected nonunion of the femur by a modified llizarov technique: follow-up study, Croat Med J 42 (6) (2001), pp. 634-641.

7. A.E. Beris, Z. Dailiana, E.O. Johnson and P.N. Soucacos, Vascularized bone grafts for the management of nonunion, Injury Int J Care Injured 375 (2006), pp. S41-S50.

8. V. Bühren, O. Gonschorek and G.O. Hofmann, Segmental transport employing intramedullary devices in tibial bone defects following trauma and infection, J Orthop Trauma 13 (3) (1999), pp. 170-177. 
9. M. Chadha, L. Maini and J. Vishwanath, The llizarov method in infected nonunion of fractures, Injury Int J Care Injured 31 (2000), pp. 509-517.

10. Y.-S. Chan, S.W.-N. Ueng and C.-J. Wang et al., Management of small infected tibial defects with antibiotic-impregnated autogenic cancellous bone grafting, J Trauma Injury Infect Crit Care 45 (October (4)) (1998), pp. 758-764.) 11. Y.-S. Chan, S.W.-N. Ueng and C.-J. Wang, Management of small infected tibial defects with antibiotic-impregnated autogenic cancellous bone grafting, $\mathrm{J}$ Trauma Injury Infect Crit Care 45 (4) (1998), pp. 758-764.

12. C.-E. Chen, J.-Y. Ko and C.-C. Pan, Results of vancomycin-impregnated cancellous bone grafting for infected tibial nonunion, Arch Orthop Trauma Surg 125 (2005), pp. 369-375.

13. S.-L. Cheng, D.C.-C. Chuang David, C.-H. Shih and S.W.-N. Ueng, Management of large infected tibial defects with radical Debride'ment and staged double-rib composite free transfer, J Trauma Injury Infect Crit Care 40 (3) (1996), pp. 345-350.

14. G. Cierny III, Infected tibial nonunion (1981-1995). The evolution of change, Clin Orthop Relat Res (March (360)) (1999), pp. 97-105.

15. G. Cierny III, J.T. Mader and J.J.A. Pennick, Clinical staging system for adult osteomyelitis. The classic, Clin Orthop Relat Res (September (414)) (2003), pp. 7-24.

16. G. Cierny III and K. Tetsworth, Osteomyelitis Debride'ment Techniques, Clin Orthop Relat Res (March (360)) (1999), pp. 87-96.

17. E. Concia, L. Massari and N. Prandini et al., Osteomyelitis: clinical update for practical guidelines, Nucl Med Commun 27 (2006), pp. 645-660.

18. L. Eralp, M. Kocaoglu and H. Rashid et al., Reconstuction of segmental bone defects due to chronic osteomyelitis with use of an external fixator and an intramedullary nail, J Bone Joint Surg 88-A (October (10)) (2006), pp. 21372145.

19. W.A. Erdman, H.T. Jayson and F. Tamburro et al., Osteomyelitis:

characteristics and pitfalls of diagnosis with MR imaging, Radiology 180 (1991), pp. 533-539.

20. I. Eshima, R.J. Feibel, K.W. Louie and D.W. Lowenberg, Combined muscle flap and llizarov reconstruction for bone and soft tissue defects, Clin Orthop Relat Res (November (332)) (1996), pp. 37-51.

21. D. Hahn and S. Olson, Surgical treatment of nonunions: a case for internal fixation., Injury Int J Care Injured 37 (2006), pp. 681-690.

22. L. Haopeng, J. Liaosha and H. Xijing, Functional development of the donor leg after vascularized fibula graft in childhood, J Paediatr Surg 35 (August (8)) (2000), pp. 1226-1229.

23. Y. Kabukcuoglu, M. kucukkaya, U. Kuzugun and M. Tezer, Management of childhood chronic osteomyelitis with the Ilizarov method, J Paediatr Orthopaed 22 (2002), pp. 632-637.

24. Y. Kobata, K. Shigematsu and H. Yajima, Vascularized fibular grafting in the treatment of methicillin-resistant Staphylococcus Aureus osteomyelitis and infected nonunion, J Reconstr Microsurg 20 (2004), pp. 1-12. 
25. A. Kocialkowski, D.R. Marsh and D.C. Shackley, Closure of the skin defect overlying infected nonunion by skin traction, Br J Plastic Surg 51 (1998), pp. 307-310.

26. M.W. Nijhof, W.J.G. Oyen and A. van Kampen, Evaluations of infections of the locomotor system with indium-111-labeled human IgG scintigraphy, J Nucl Med 38 (1997), pp. 1300-1305.

27. M. Panagiotis, Classification of nonunion, Injury Int Care Injured 365 (2005), pp. S30-S37.

28. M.J. Patzakis and C.G. Zalavras, Chronic posttraumatic osteomyelitis and infected nonunion of the tibia, J Am Acad Orthop Surg 13 (October (6)) (2005), pp. 417-427. 